\title{
CITYGML AND THE STREETS OF NEW YORK - A PROPOSAL FOR DETAILED STREET SPACE MODELLING
}

\author{
C. Beil \& T. H. Kolbe
}

Chair of Geoinformatics, Technical University of Munich, Germany - (christof.beil, thomas.kolbe)@tum.de

KEY WORDS: CityGML, 3D City Models, Street Space, Roads, Transportation, OpenDrive, LandInfra, New York City

\begin{abstract}
:
Three-dimensional semantic city models are increasingly used for the analysis of large urban areas. Until now the focus has mostly been on buildings. Nonetheless many applications could also benefit from detailed models of public street space for further analysis. However, there are only few guidelines for representing roads within city models. Therefore, related standards dealing with street modelling are examined and discussed. Nearly all street representations are based on linear abstractions. However, there are many use cases that require or would benefit from the detailed geometrical and semantic representation of street space. A variety of potential applications for detailed street space models are presented. Subsequently, based on related standards as well as on user requirements, a concept for a CityGML-compliant representation of street space in multiple levels of detail is developed. In the course of this process, the CityGML Transportation model of the currently valid OGC standard CityGML2.0 is examined to discover possibilities for further developments. Moreover, a number of improvements are presented. Finally, based on open data sources, the proposed concept is implemented within a semantic 3D city model of New York City generating a detailed 3D street space model for the entire city. As a result, 11 thematic classes, such as roadbeds, sidewalks or traffic islands are generated and enriched with a large number of thematic attributes.
\end{abstract}

\section{INTRODUCTION}

Semantic 3D city models are experiencing a veritable boom and often are the foundation for a wide range of analysis and simulations. These models allow the accurate representation of landscapes as well as urban areas and are therefore interesting for a variety of applications. Until now the focus has mostly been on models of buildings. Firstly because of their dominant role within the cityscape and secondly because of the lack of information and data sources for other thematic areas such as street space. This however has changed in recent years. A rising number of cities and communities provide information about streets by single line representations. In some cases, even areal data on the exact shape of street space objects is available. This development is fairly new, therefore there are very few guidelines on detailed areal representations of roads and street space within city models, let alone actual implementations. Most existing standards focus on a linear or parametric representation of roads. This leads to some problems if a detailed and highly accurate areal model of street space is required. Continuing a study project conducted by the Chair of Geoinformatics of the Technical University of Munich (see Kolbe et al. 2015), this work explores the possibility for a detailed generation of street space objects within semantic 3D city models based on open data and compliant to the CityGML standard. The aim of this paper is to illustrate how potential applications would benefit from accurate street space models. Subsequently a representation concept of such a model based on modelling requirements is developed and selected parts of this concept are implemented within a semantic 3D model of New York City.

\section{RELATED STANDARDS}

There are several standards and guidelines dealing with possibilities to represent streets within different kinds of models. Most of these standards focus on a single line or parametric representation of streets. Nonetheless they contain elaborate concepts and ideas on how to represent road networks and thus should be examined and discussed. Some of the most commonly used and most relevant standards and guidelines are presented in the following section of this article.

\subsection{Geographic Data Files}

Geographic Data Files (GDF) is a standard mainly used in vehicle navigation for the exchange of digital maps between map manufacturers and navigation system integrators. Additionally, GDF provides numerous rules for data capture and representation, in regard to many street space objects. The current specification GDF5.0 was published in 2011 by ISO and is divided into several sections (ISO 14825, 2011). Real world objects called 'Features' and their database representation form the core of the general GDF data model. Furthermore, topological and non-topological relations between these features as well as their characteristics are described. Additionally, these features are organized semantically by categorizing them into different thematic feature themes defined in the so-called Feature Catalogue. Possible attributes and relationships of these features are specified in the corresponding attribute catalogue and relationship catalogue. Features can also be subdivided based on the topology of the underlying graph by which the features are defined. Moreover, all objects can be conceptually divided over three levels called Level-0, Level-1 and Level-2. This concept shall be illustrated by explaining the most relevant thematic category with regard to street space called 'Roads and Ferries'. This contains a so called 'Road Network' which can be represented in Level-1 and Level-2 respectively. A representation in Level-0 would consist of basic topological building blocks such as nodes, edges or faces but is not available for road network representations. Simple features such as 'Road Elements' or 'Junctions' make up Level-1 objects and can be aggregated to form more complex features called 'Road' or 'Intersection' in Level-2. Road networks are represented by linear structures. Enclosed traffic areas, defined as areas within unstructured traffic movements such as car parks, can be represented as areas. GDF supports a mechanism of linearly referencing points along line features. Furthermore, all geodetic parameters needed for the correct interpretation of $\mathrm{X}, \mathrm{Y}$ and $\mathrm{Z}$ values shall be described explicitly to make a transformation into any other coordinate system possible. While many basic concepts of GDF are explained using UML diagrams, there is also a complete XML schema defined within the standard document. 


\subsection{ASB and OKSTRA}

'Anweisung Straßeninformationsbank' (ASB) and 'Objektkatalog für Straßen- und Verkehrswesen' (OKSTRA) released by the Federal Ministry of Transport and Digital Infrastructure of Germany, are standardized catalogues for the uniform recording, manipulation and provision of street object characteristics (BASt, 2016). ASB describes object structures from a technical perspective, whereas OKSTRA focuses on formal descriptions using data schemas and UML representations of street space objects (BMVI, 2014). The concept is described using definitions given in ASB. First, types of streets that should be included are designated. A linear representation is used to illustrate the described modelling concept. Every street is divided into several sections with each section being bounded by 2 uniquely identified nodes. Every section inherits a stationing system, starting at the first node and ending at the second. A node can be made up of multiple smaller branches like ramps or driveways connecting different sections. The standard proceeds by giving numerous detailed examples, again illustrated via line and node representations, on how to represent various street space scenarios. These include inter alia intersections in different levels of complexity, roundabouts, bridges, and overpasses.

\subsection{OpenDrive}

OpenDrive is an open data format to describe street networks and is commonly used for driving simulations. The current format specification, rev. 1.4 was published in 2015 by VIRES Simulationstechnologie GmbH. The two most frequently used coordinate systems within the standard are an inertial, right handed coordinate system according to ISO 8855 and a so-called track system that applies along a defined reference line. All roads are represented using a reference line described as a sequence of geometric primitives such as straight lines, spiral curves, cubic polynoms and parametric cubic curves. Along this reference line a variety of road properties such as an elevation profile, traffic signs or lanes can be defined. Multiple 'Lane Sections' with a constant number of lanes along a reference line may be defined in ascending order. The properties of each lane, however, may vary and are defined relative to the start of the corresponding lane section (Dupuis et al. 2015). Additionally, a road's superelevation crossfall as well as a lateral profile can be modelled. Lanes are identified by a unique number per lane section and ordered in sequence starting from 0 , ascending to the left and descending to the right (relative to the reference line direction). The number of lanes is unlimited and the reference line itself is defined as lane 0 with width zero. In some situations, a 'Lane Offset' can be introduced in order to shift the lane profile. This can be useful to describe a turning lane by offsetting lane 0 using a cubic polynom. To make navigation through a road network possible, roads are linked either by a successor/predecessor linkage or via junctions. If the linkage between two roads is clear a standard link can be used. Junctions are required if an incoming road could be linked to more than one successive road. OpenDrive provides two approaches to describe surfaces parametrically. The standard description contains parameters per lane for 'surface material code', 'roughness' and 'friction'. More detailed information on road surface data may be included in an extended description. Road networks may be geo referenced using a projection definition formatted as 'proj4'string. A complete XML schema definition for OpenDrive XML files can be examined on the standards webpage ${ }^{1}$.

\subsection{LandInfra}

The OGC standard 'Land and Infrastructure Conceptual Model Standard' (LandInfra) defines concepts for providing and understanding information about land and civil engineering infrastructure (Gruler et al. 2016). These concepts are formally defined using UML diagrams. The standard covers various subject areas defined by so called requirement classes. The most relevant in terms of street modelling are Alignment and Road. An Alignment is defined as a positioning element which provides a linear referencing system for locating physical elements. It can be defined in several ways such as horizontal, vertical or $3 \mathrm{D}$ alignment. For roads, there is typically an Alignment for the centreline. For dual carriageway roads, separate alignments should be realised, however they may also share a reference horizontal alignment at the approximate centre of the entire road. Based on a Linear Referencing Method (LRM) locations along the Alignment can be defined as linearly referenced locations. DistanceAlong and offsetLateralDistance values shall be measured in the horizontal plane and ignore any vertical displacement if Alignment is used as a linear element. OffsetVerticalDistance values can be considered. The class Road contains multiple alternatives for representing a road design such as RoadElements, $3 D$ StringLines ( $\sim$ profile views), $2 D$ CrossSections and '3D surfaces and layers'. Road elements can include many different types such as pavement, sidewalk or curb defined by an attribute called RoadElementType. Multiple road elements can be grouped together as RoadElementSet. RoadElements can be physically located optionally by a spatial representation or a linearly referenced location. Any particular point in a cross section can be represented by a 3DStringLine. The surface of a road can be represented by a triangulated irregular network (TIN). This is not limited to the top surface of the constructed road but can also be used to model subsurface information.

\subsection{OpenStreetMap}

OpenStreetMap is a project that provides user-generated maps available for web viewing and download (Haklay \& Weber 2008). Map features defined on the project homepage ${ }^{2}$ are identified by so called keys. These include 'highway=*' used for any kind of road, street or path. An assigned attribute value further indicates the importance of each highway within the road network. Streets are represented as linear structures, often enriched with information on number of lanes, speed limits, or street names.

\subsection{Discussion of the standards presented}

GDF, OKSTRA and ASB offer extensive regulations on dividing street space objects into different categories. OpenDrive, ASB and LandInfra deliver solutions for problems not addressed in the current CityGML standard like linear referencing and stationing systems. With the exception of LandInfra the presented standards focus on linear and parametric representations of streets only. This can lead to some problems if a detailed representation of streets and street space objects is required as illustrated in figure 1 . While a linear representation typically is sufficient for applications like navigation, traffic simulations or noise mapping, often an areal street model is needed in order to represent geometric details like bus stops, irregularly changing street widths or road markings. Additionally, large sealed surfaces like plazas or parking lots should be modelled as surface

\footnotetext{
${ }^{2}$ https://wiki.openstreetmap.org/wiki/Main_Page
}

\footnotetext{
${ }^{1}$ http://www.opendrive.org/tools/OpenDRIVE_1.4H.xsd
} 
geometries. The following paragraph discusses a number of potential applications for detailed street space models and explains how these would benefit from surface-based representations.

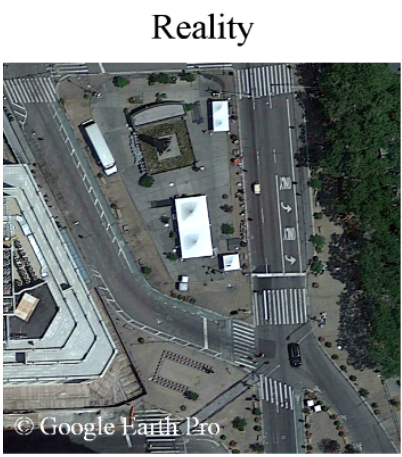

Linear representation

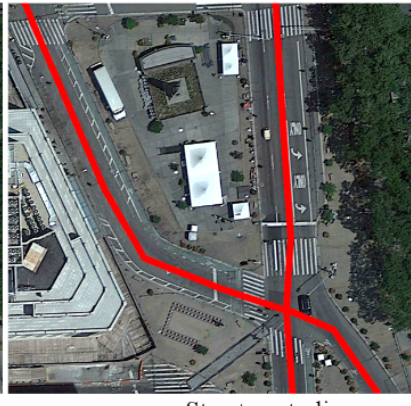

Parametric representation

Areal representation

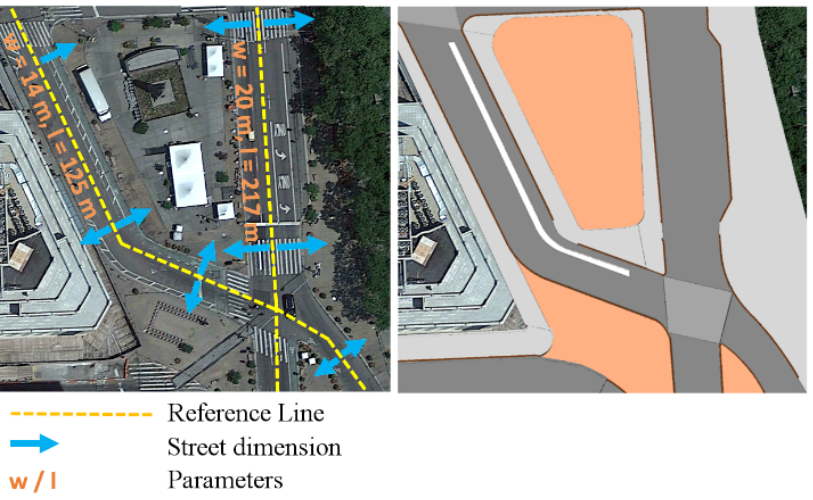

Figure 1. Comparison of different representation types

\section{APPLICATIONS FOR DETAILED STREET SPACE MODELS}

Detailed areal representations of street space are useful for a variety of different applications such as autonomous driving, driving simulators or emergency planning. In order to illustrate this claim the following section examines possible applications and describes how each one of them would benefit from detailed $3 \mathrm{D}$ areal street space models. Furthermore, the modelling requirements are presented from a user perspective.

\subsection{Potential fields of application}

\subsubsection{Infrastructure planning and management}

Digital 3D city models can be the basis for land use management (Ross 2010). Besides settled areas the cityscape is mainly shaped by public traffic areas. Visual simulations of constructed areas as well as free space can be used in order to plan different scenarios and conduct effort and cost analysis. In this context, large construction projects such as new highway sections or bridges can be planned digitally. This can also be used to visualize the future 3D view of these constructions and thus prevent resistance of the citizens. Many communities have a duty to fulfill their municipal obligations such as clearing streets of snow or leaves or taking care of sufficient street lighting. Planning these often very expensive tasks in order to find the most effective and economic practical implementation could be supported by detailed street space models. This also involves maintenance of streets and damage mapping. Areal street models combined with knowledge of pavement conditions can be used for assessments of expected repair costs. Kolbe et al. (2008) showed how
CityGML and sematic city models can be used for emergency planning. In this context, Rupprecht et al. (2011) described ways to conduct simulations of pedestrian flows. Such simulations need the exact 3D representation of navigable surfaces to identify bottle-necks.

\subsubsection{Spatial analysis}

Detailed 3D street space models can be the foundation for a variety of spatial analysis methods. Local heat islands in largely sealed areas such as street intersections or plazas can be analyzed using information derived from areal street representations in combination with knowledge of solar irradiation. The optimal placement of street signs and traffic lights can be planned within city models and supported by visibility analysis. Ghassoun et al. (2014) showed how city models can be useful for air quality analysis. Parameters such as number of intersecting streets, their respective width and angle between street arms can be derived from accurate street space models. In combination with other parts of a city model like buildings and vegetation highly accurate simulations can be conducted. Based on areal information of streets and footpaths clearance spaces can be easily modelled. These can be used to simulate a heavy-load transportation and identify problematic areas. While linear representations of streets are often sufficient for noise simulations, areal models can be used to visualize the results. Detailed street models with information on elevation can also be the foundation for water runoff simulations with high levels of detail.

\subsubsection{Automotive related applications}

Knowledge on the exact shape of street space objects can be of high use for autonomous driving applications. StrassenburgKleciak (2016) stated that information on street edges can be used in order to increase driving safety. Connected vehicles in combination with data on length and width of certain street sections can be used to assist drivers with overtaking manoeuvres. Randt et al. (2007) described how virtual 3D landscapes can be used for driving simulators and emergency driver training. Other automotive related simulations such as traffic simulations or driving dynamic simulations (see Butz et al. 2004) can also be supported by information derived from detailed street space models. Roads normally forbidden for automobiles but wide enough to be used by ambulances in emergency situations could be integrated into navigation systems. In this context knowledge on steps, curbs, etc. could be considered for barrier free route planning in order to assist persons with reduced mobility.

\subsection{General modelling requirements}

The presented applications impose certain demands on how street space should be modelled. These modelling requirements can be divided into the following categories.

1. 3D positional accuracy: Relative or absolute accuracy with which object coordinates are determined.

2. Geometric resolution: The degree to which geometric details of individual objects are represented.

3. Thematic accuracy: Describes the possibility to distinguish between different thematic objects.

4. Topicality: For most applications it is important to have an up-to-date model of a real world scenario.

5. Network and areal topology: Topological relations between linear and areal representations of road networks or street space objects.

6. Dynamic component: Representing and switching between models in different stages of development. 
7. Visualization: Describes the importance to visualize objects realistically by employing suitable colouring or textures.

Beil (2017) conducts a more detailed analysis on potential applications as well as their requirements with regard to detailed street space models. Biljecki et al. (2015) discussed a huge variety of further applications of virtual $3 \mathrm{D}$ city models in general. Tamminga et al. (2013) analyzed modelling requirements focusing on traffic and transportation models and also discussed possibilities to match these requirements to the CityGML data structure.

Based on the applications presented above as well as on their requirements for detailed street space models, the currently valid OGC standard CityGML2.0 is examined for potential deficits and improvements to its 'Transportation Model' are proposed.

\section{MODELLING STREETSPACE WITH CITYGML}

City Geography Markup Language (CityGML) is an open data model and XML-based format to represent, store, and exchange semantic 3D city and landscape models. The current standard CityGML 2.0 was issued by the Open Geospatial Consortium (OGC) in 2012. It defines numerous classes and relations for many thematic city objects with respect to their spatial, semantic, and appearance properties. All city objects can be represented in 5 consecutive Levels of Detail (LoD) (Gröger et al. 2012). The most relevant thematic model in the context of representing street space is the 'Transportation Model'. It consists of the main class TransportationComplex and can be thematically specialized into 4 subclasses called Road, Square, Track, and Railway. Transportation features can be represented as linear networks in LoD0. Starting from LoD1 transportation objects are spatially represented by (multi)surfaces. LoD2-LoD4 representations allow a further semantic decomposition into TrafficAreas (e.g. driving lanes or sidewalks) and objects not directly used for vehicle or pedestrian movement called AuxiliaryTrafficAreas (e.g. middle lanes, kerbstones or green space). Attribute values for properties like class, function, or usage are defined using code lists enumerating the specific possible entries.

\subsection{Deficits of the CityGML2.0 Transportation Model}

The 'Transportation Model' in CityGML2.0 is mainly designed to define a basic framework for a uniform modelling concept of street space objects and is intended to complement and not to replace existing standards such as GDF. Nevertheless, some deficits of the current 'Transportation Model' can be identified.

\subsubsection{Insufficient specifications}

1. It is unclear which axis should be used to represent streets in LoD0 (roadway- or lane-axis).

2. The possibility to represent intersections as nodes in LoD0 is implied by the defined geometry class GeometricComplex but not specified otherwise.

3. Roads modelled in LoD1 are to be represented by MultiSurface geometries. It is not clear which objects of street space should be included in this representation.

4. The segmentation of a TransportationComplex into multiple Road, Track, Square, or Railway objects is possible but not specified any further. Until now it is possible to represent the entire street space of a city by just one Road object.

5. Intersections and roundabouts are not represented explicitly.

\subsubsection{Missing stationing / linear referencing concept}

Attributes like speed limits can change in the course of a road. In order to represent these changes the corresponding object would have to be split in smaller parts with individual attributes. This is inconvenient and impractical. Sometimes attributes like speed limits may vary time dependent or may depend on symbols shown on sign gantries. This problem could be solved by introducing a stationing or linear referencing system. Attributes could then be specified for certain sections of a transportation object without having to split its geometry.

\subsubsection{Other points of criticism}

1. An additional class RoadSection could define a clear segmentation concept for road network objects.

2. It is not clear if TrafficAreas representing parking lots should be part of the subclass Road or Square.

3. The graphic used in the CityGML2.0 standard to illustrate Squares can be misinterpreted. The displayed intersection should be part of the subclass Road and not Square. Squares should be defined as plazas mainly used by pedestrians or large areas with sealed surfaces like parking lots.

4. Intersections belong to multiple streets. It is not clear how to model this circumstance while avoiding a redundant representation.

5. The main difference between LoD3 and LoD4 models is the possibility to represent interior structures of buildings such as individual rooms. For many other thematic models, including the 'Transportation Model', this can lead to absurd results.

6. Subsurface structures such as material layers are not considered yet.

7. Clearance spaces could be represented by extruding road- or pathway footprints. This is not specified yet.

8. Geometric and semantic subtleties such as manholes, drains or roadway damages are not defined.

\subsection{Proposal for a revised Transportation Model}

These points of criticism as well as potential user requirements serve as inspiration for a proposal of a revised 'Transportation Model'.

\subsubsection{Data Model}

First an advanced data model of the CityGML 'Transportation Model' is presented. The basic structure of this model with TransportationComplex as main class divided into 4 subclasses Road, Square, Track, and Railway remains in place. From LoD2 newly labelled TrafficSpaces and AuxiliaryTrafficSpaces can be spatially modelled by MultiSurface as well as MultiSolid geometries. Furthermore, linear representations of street space should not be limited to LoD0. By specifying which axis of a street should be used, linear representation with different levels of complexity become possible. Squares are defined as large sealed surfaces such as plazas or parking lots. By introducing a new class called Section a segmentation of Road, Track, and Railway objects is possible and further specified using a class attribute with corresponding code list values. Moreover, representations of subtle objects such as manholes or roadway damages can be allowed as a LoD3 representation. Clearance space models in the form of solid geometries representing free space above traffic surfaces are proposed together with a corresponding code list defining possible class attribute values. The usage of XLinks avoids the redundant representation of street sections that are part of multiple streets in CityGML instance documents. This data model with newly introduced classes, attributes, geometries as well as a revised LoD concept is shown as UML diagram in figure 2 and explained in detail in the following subsections. 


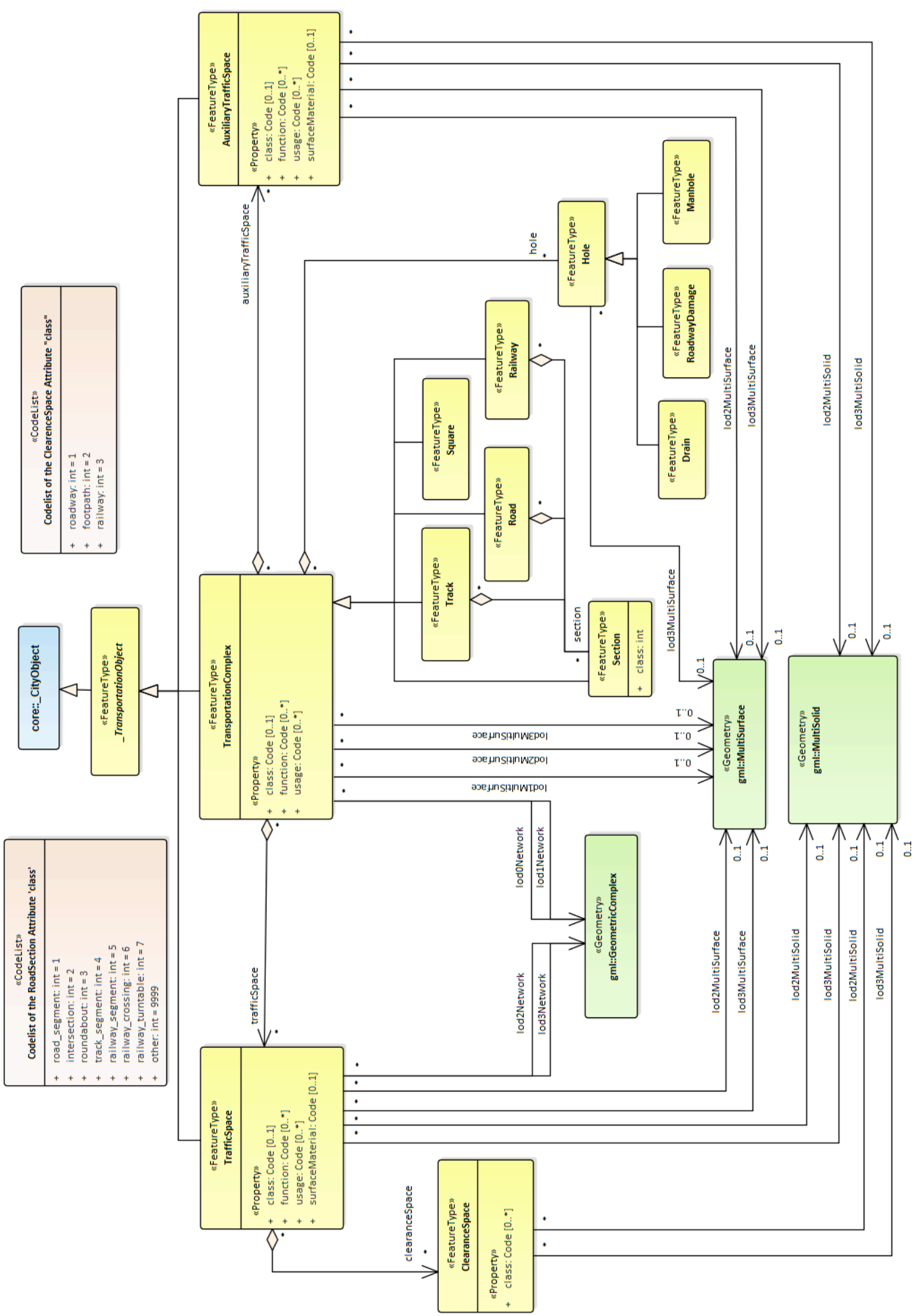


Figure 2. UML diagram of the improved CityGML Transportation Model

\subsubsection{Four Levels of Detail instead of Five}

Löwner et al. (2016) propose a new LoD concept representing all city features in just four levels of detail. Following this idea, it is proposed to model street space objects of the 'Transportation Model' in LoD0-LoD3. Figure 3 shows a 'real world' street segment with multiple thematic parts. It is indicated that an areal LoD1 representation should contain the entire width of the street, including sidewalks, kerbstones or barriers. A more detailed segmentation into TrafficSpaces and AuxiliaryTrafficSpaces should be realised starting from LoD2 while LoD3 additionally allows the representation of subtle structures such as manholes or roadway damages. This is very similar to the way how building models are represented thematically more detailed in CityGML with increasing LoD.

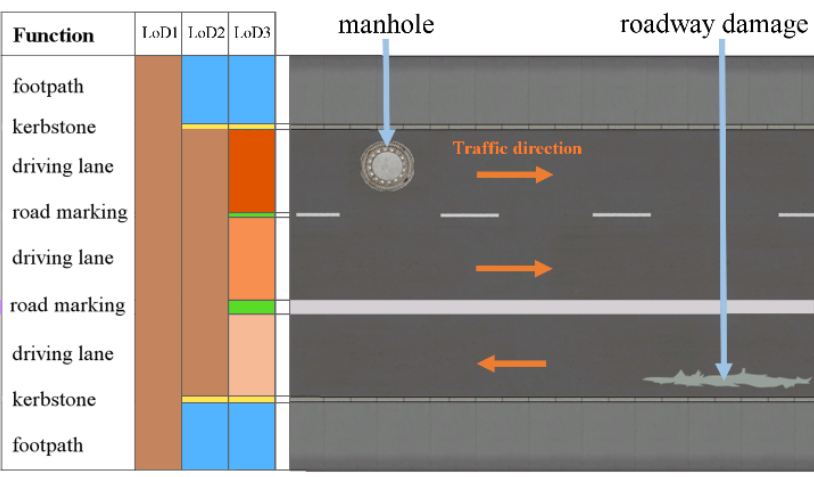

Figure 3. Street section in LoD1-3

In addition to areal models, figure 4 further specifies which street axis should be used for linear representations in each LoD and also gives an example for the representation of roadway damages in LoD3. These holes in a street surface can extend over more than one road section and therefore should be modelled as a separate class (and not as a part of a Section) and spatially represented by MultiSurface geometries.

LoD0

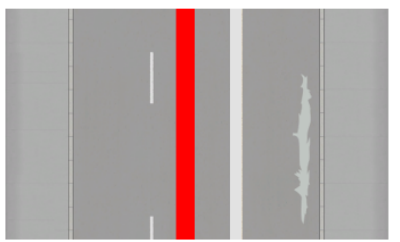

LoD2

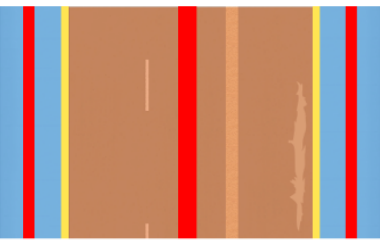

Linear representation

manumation

Figure 4. Linear and areal representations in LoD0-3

As long as roadways are not topologically separated, streets are represented by a single centreline per driveway in LoD0 and LoD1. Starting from LoD1, street space can be modelled by linear and/or areal objects respectively. In addition to the driveway centreline, linear representations for footpaths and bikeways become possible in LoD2, thus enabling a more detailed thematic decomposition of street space. While driveways are still represented by a single TrafficSpace object in LoD2, LoD3 representations finally contain one TrafficSpace object for each individual driving lane. Figure 5 shows a direct comparison of linear network representations in LoD0 and LoD3. While lod0Netwoks are modelled with one axis per driveway and section (defined in the following paragraph) connected with nodes representing intersections, lod3Networks contain separate lines for each individual driving lane, following every possible way a car could take. This automatically implies a predecessor/ successor concept useful for some applications.
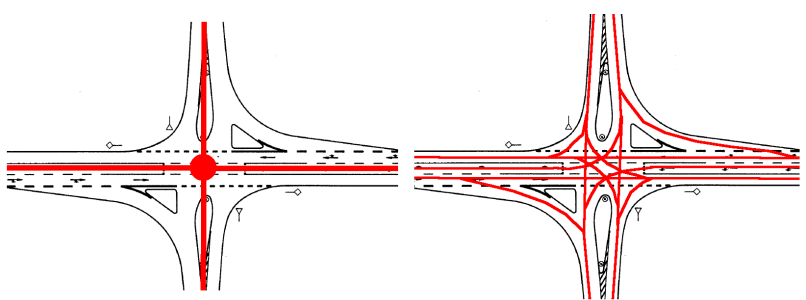

Figure 5. Comparison of the lod0Network and lod3Network representation of an intersection

\subsubsection{Introducing sections}

A proposed concept to divide street networks into several sections is illustrated in figure 6 .

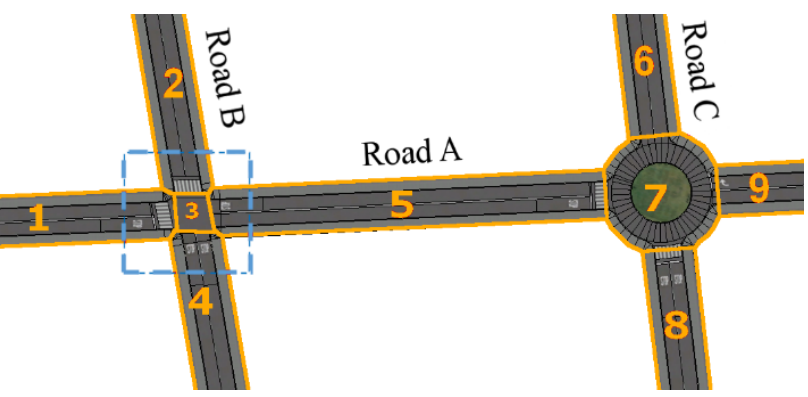

Figure 6. Illustration of road sections

Road objects representing an entire street could be split into 3 different types of sections declared by class attribute values defined in a corresponding code list. These types are 'road_segments' (Nr. 1, 2, 4, 5, 6, 8 and 9), 'intersections' (Nr. 3) and 'roundabouts' (Nr. 7). In the given example Road A consists of Sections Nr. 1, 3, 5, 7 and 9 while Road B is composed of Sections Nr. 2, 4, and also 3. This means Road A and Road B share Section 3. In order to avoid a redundant representation of this shared object, XLinks are used in the CityGML instance document to reference the shared section. This is visualized in figure 7 .

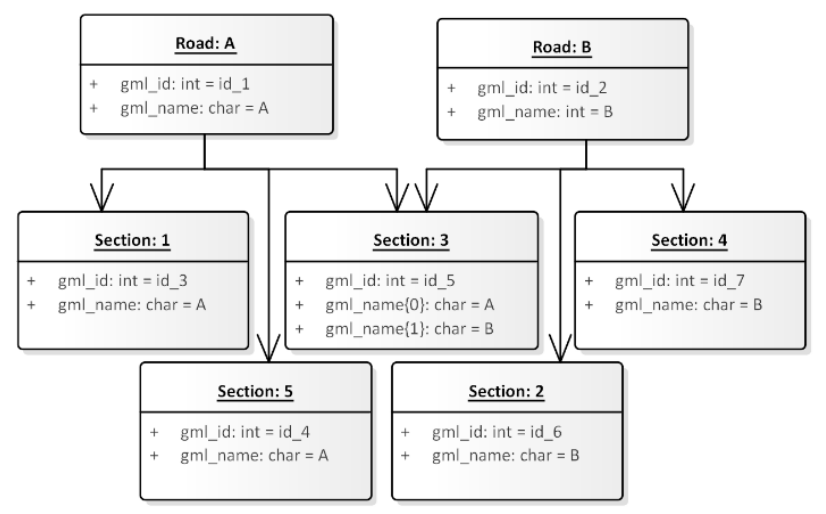


Figure 7. Instance diagram illustrating the referencing of a section shared by two roads

Usage of the XLink concept to avoid redundant representations is explained in more detail in the CityGML specification document (Gröger et al. 2012).

\section{IMPLEMENTATION FOR NEW YORK CITY}

The detailed representation of the street space is tested using the example of New York City. The NYC Open Data Portal provides an extensive number of datasets, including geometric as well as semantic information on street space objects for the entire city suitable for detailed street space modelling.

\subsection{Data sources}

There are three main data sources used to generate a detailed streets space model of New York City. Within a study project conducted by the Chair of Geoinformatics of the Technical University of Munich a semantic 3D city model of New York City was generated (Kolbe et al. 2015). The produced CityGML datasets are available for download on the project website ${ }^{3}$.

The provided road dataset includes a LoD0 line-network representation and is the basis for the street model generated in this work. The second major data source is the so called 'NYC Planimetric Database' provided in the New York City Open Data Portal. This contains representations of a variety of features such as roadbeds, sidewalks, or parking lots in the form of areal Shapefile data. Additional data is gathered searching websites of the NYC Department of Transportation and the NYCD of City planning. This includes information such as speed limits and pavement ratings as well as guidelines with respect to the physical dimensions and used materials of street space objects described in the so called 'NYC Street Design Manual'.

\subsection{Implementation}

All data transformations and manipulations were performed using the Software 'Feature Manipulation Engine' (FME 2016.1). For the storage, management and integration of the large amounts of data generated the open source geodatabase $3 D C i t y D B$ Version 3.3 was employed. The actual implementation had to be based on the currently valid CityGML2.0 Transportation Model but already considered suggestions made in the previous chapter of this article.

\subsubsection{Data preparation}

First, the data provided by the 'Planimetric Database' had to be transformed into the coordinate reference system (EPSG:32118) used by the mentioned project. Second, suitable test areas were selected in order to try out different implementation approaches with a manageable amount of data. Also very complex scenarios such as highway interchanges had to be filtered out and modelled separately. After these preparations, the input data consisted of street centrelines with a large number of attributes, additional datasets containing further attributes for each centreline segment and accurate areal data on multiple thematic features such as roadbeds, sidewalks or traffic islands (Planimetric Database). The main tasks are to integrate these data collections into one CityGML compliant dataset.

\subsubsection{Data enrichment}

Additional attributes like speed limits, pavement ratings, or number of lanes from mentioned datasets were matched to the already CityGML compliant centreline data via corresponding

\footnotetext{
${ }^{3}$ https://www.gis.bgu.tum.de/projekte/new-york-city-3d
}

attributes such as 'street name' or 'segment ID'. The information contained in this 'new' centrelines dataset then was transferred to corresponding areal roadbed geometries using a spatial matching method. All available street space objects like sidewalks, parking lots etc. then where manipulated semantically and geometrically in order to achieve a detailed semantic 3D street space model of the entire city. Specifications concerning physical dimensions such as sidewalk heights etc. were implemented based on specifications provided by mentioned 'NYC Street Design Manual'. Due to the huge amount of data multiple thematically divided CityGML files were generated for each street space object class respectively. These include 11 thematic object classes with a total of 508,660 objects, each one assigned to the most fitting of the 3 possible subclasses Road, Square, or Track. The CityGML datasets generated were further processed in two different ways. Firstly, using 3DCityDB KML-/COLLADA-files as well as corresponding spreadsheets were generated for each thematic class individually in order to integrate semantic and geometric information in terms of a tiled KML visualization model. This was achieved using the '3D-Web-Map-Client' (see (Chaturvedi et al. 2015), (Kolbe et al. 2016)) and visualized with the open source 'WebGL Virtual Globe Cesium'.

Secondly, a smaller area was selected and enhanced with suitable textures to accomplish a more realistic visualization. Also, the data structure of this excerpt was adjusted by introducing TrafficAreas and AuxiliaryTrafficAreas, thus further specifying individual street space objects and generating a LoD2 street space model. In order to express affiliations to top-level features each object was linked to superordinated Road, Square, or Track objects. Being based on CityGML2.0, the section concept introduced earlier could not be implemented explicitly yet but was already taken into account by creating individual Road objects for each section based on the suggestions made in chapter 4 , thus proving the practicability of the concept. More detailed information on the actual implementation including the FME workbenches can be found on the project's GitHub page ${ }^{4}$.

\subsection{Results}

Figure 8 illustrates the generated detailed 3D street space model in LoD2. The upper image is a screenshot of the mentioned 3D Web-Map-Client implemented for the entire city showing the area around Columbus Circle south west of Central Park. The lower image displays an excerpt of the generated 3D model showing an intersection with textured roadbeds as well as raised sidewalks, curbs, and traffic islands. Among other improvements, intersections are represented as separate, non-overlapping features containing information on all intersecting street names.

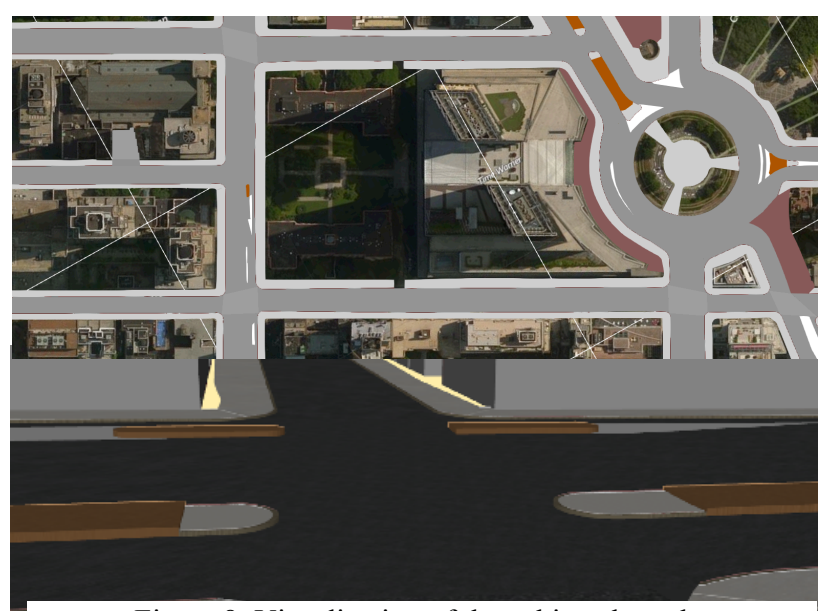

Figure 8. Visualization of the achieved results

${ }^{4}$ https://github.com/tum-gis/3d-model-new-york-city 
Please note, that this road space model is not the result of a procedural generation, but reflects the exact layout of the real street space of NYC. Hence, it can be used for quantity take-off (e.g. paved surface area of Broadway) or measurement tasks.

\section{CONCLUSION AND OUTLOOK}

This paper discussed a range of applications which require the detailed 3D representation of the street space. It was shown that most existing standards on street space modelling provide linear and parametric representations, mostly in $2 \mathrm{D}$, only. In order to cover the specific use case requirements, extensions and improvements to the CityGML Transportation Model for the detailed spatio-semantic representation of the street space were made. In the new model, each LoD can have a center line representation, a surface based representation, or both. While LoD0 consists of just a generalized linear network, LoD1 augments this by the representation of the 3D surface geometry. In LoD2 the street surfaces are partitioned into roadbed, pedestrian walkway etc. In LoD3 even the individual driving lanes are semantically and spatially separated making these datasets usable for vehicle navigation and detailed traffic simulations. In LoD3, TransportationComplexes can have holes, e. g. to model gully openings, road damages, or manholes. Road sections are represented explicitly allowing to model a long road as one object consisting of sections which are sub classified into road segments, intersections, and roundabouts. Furthermore, clearance space above the traffic surfaces can be represented by 3D solids. The concepts are illustrated by the detailed representation of the street space for New York City. This 3D model was generated using multiple datasets from the NYC Open Data store and is provided in CityGML on the project homepage. In the future, the data model should be further extended by a linear referencing method. Also, a concept for representing zones for speed limits etc. could be added. Currently, we are also working on using the NYC 3D street model to derive input datasets for the micro traffic simulation software VISSIM.

\section{REFERENCES}

BASt, 2016. Objektkatalog für das Straßenwesen, Version 2.017. Bundesanstalt für Straßenwesen. http://www.okstra.de/docs/2017/okstra_2017.pdf (March 2017)

Beil, C., 2017. Detaillierte Repräsentation des Straßenraums in 3D-Stadtmodellen. Technical University of Munich, Chair of Geoinformatics, Master's Thesis (in German).

https://mediatum.ub.tum.de/node?id=1350734 (March 2017)

Biljecki, F., Stoter, J., Ledoux, H., Zlatanova, S., \& Çöltekin, A., 2015. Applications of 3D city models: State of the art review. ISPRS International Journal of Geo-Information, 4(4), 28422889.

BMVI, 2014. Anweisung Straßeninformationsbank Kernsystem Version 2.03. Bundesministerium für Verkehr und digitale Infrastruktur. http://www.bast.de/DE/Verkehrstechnik/Publikati onen/Regelwerke/V-asb-kernsystem.pdf (March 2017).

Butz, T., Ehmann, M., Stryk, O.v., Wolter, T-.M., 2004. Realistic road modelling for the real-time simulation of vehicle dynamics In: ATZ worldwide 106.2: 11-13.

Chaturvedi, K., Yao, Z., Kolbe, T.H, 2015. Web-based Exploration of and Interaction with Large and Deeply Structured Semantic 3D City Models using HTML5 and WebGL In: Wissenschaftlich-Technische Jahrestagung der DGPF und Workshop on Laser Scanning Applications. Vol. 3.
Dupuis, M., Bahram, M., Grezlikowski, H., Richter, A., Goldberg, A., Klaerner, E., Bock, B. Kaellgren, L., 2015. OpenDrive - Format Specification, Rev. 1.4, VIRES Simulationstechnologies GmbH. http://www.opendrive.org/

Ghassoun, Y.; Löwner, M-.O., Weber, S., 2015. Exploring the benefits of $3 \mathrm{D}$ city models in the field of urban particles distribution modelling - A comparison of model results. In Advances in 3D Geo-Information Sciences; Springer, Berlin, pp. 193-205.

Gröger. G., Kolbe, T. H., Nagel, C., Häfele, K-.H., 2012. OGC City Geography Markup Language (CityGML) Encoding Standard, OGC 12-019.

Gruler, H-.C., Stubkjaer, E., Axelsson, P., Wikstrom, L., 2016. OGC Land and Infrastructure Conceptual Model Standard (LandInfra), OGC 15-111.

Haklay, M., Weber, P., 2008. OpenStreetMap User-generated street maps. IEEE Pervasive Computing 7 (4): 12-18.

ISO 14825, 2011. Intelligent transport systems - Geographic Data Files (GDF) - GDF5.0. International Standard, ISO.

Kolbe, T. H., Gröger, G., Plümer, L., 2008. CityGML - 3D City Models and their Potential for Emergency Response. In: Zlatanova, Li (eds.), Geospatial Information Technology for Emergency Response, Taylor \& Francis.

Kolbe, T. H., Burger, B., Cantzler, B., 2015. CityGML goes to Broadway. In: Photogrammetric Week'15, Wichmann.

Kolbe T. H., Yao, Z., Nagel, C., Redweik, R., Willkomm, P., Hurda, G., Müftüoglu, A., Kunde, F., 2016. 3D City Database for CityGML Version 3.3.0 Documentation. www.3dcitydb.org

Löwner, M-.O., Gröger, G., Benner, J., F. Biljecki, C., Nagel, 2016. Proposal for a new LoD and multi-representation concept for CityGML. In: ISPRS Annals of the Photogrammetry, Remote Sensing and Spatial Information Sciences, Vol. IV-2/W1, 11th 3DGeoinfo Conference in Athens, Greece.

Randt, B., Bildstein, F., Kolbe, T. H., 2007. Use of Virtual 3D Landscapes for Emergency Driver Training. In: Proc. of the Int. Conference on Visual Simulation IMAGE in Scottsdale, Arizona.

Ross, L., 2010. Virtual 3D City Models in Urban Land Management - Technologies and Applications. Ph.D. Thesis, Technische Universität Berlin, Germany.

Rupprecht, T., Klingsch, W., Seyfried, A., 2011. Influence of geometry parameters on pedestrian flow through bottleneck, in Pedestrian and Evacuation Dynamics 2010. In: Peacock, R., Kuligowski, E., Averill, J. (Ed.). Springer, US, 71-80.

Strassenburg-Kleciak, M., 2016. OpenStreetMap - Straßen als Flächen erfassen. In: gis.Business 2/2016, Wichmann. See also http://wiki.openstreetmap.org/wiki/Proposed_features/Street_ar ea (March 2017)

Tamminga, G., van den Brink, L., van Lint, H., Stoter, J., Hogendoorn, S., 2013. Towards GIS-Compliant Data Structures for Traffic and Transportation Models. In: Transportation Research Board 92 ${ }^{\text {nd }}$ Annual Meeting in Washington D.C. 